\title{
Evaluation of a computer-based nutrition education tool
}

\author{
Katrin Kreisel ${ }^{1,2, *}$ \\ ${ }^{1}$ Institute of Nutritional Sciences, University of Vienna, Austria: ${ }^{2}$ Present affiliation: Korean Institute for Health \\ and Social Affairs, Seoul, Republic of Korea
}

Submitted 28 April 2003: Accepted 31 July 2003

\begin{abstract}
Objective: To evaluate the efficacy and feasibility of using a computer-based teaching tool (http://www.coolfoodplanet.org) for nutrition and lifestyle education developed for primary school children.

Design: This was a 2-week school-based intervention in third and fourth grades. The study design was multi-factorial with repeated measures of nutrition knowledge, at three points in time, of dependent samples from control and intervention groups. Control schools $(n=7)$ used 'traditional' nutrition education materials and intervention schools $(n=8)$ additionally used the computer-based educational tool. Qualitative information was collected in focus group discussions with student teachers and pupils, and by observing the nutrition lessons.

Setting/subjects: Pupils aged $8-11$ years $(n=271)$ from participating schools in Vienna, Austria.

Results: Nutrition knowledge increased significantly in both intervention and control schools, irrespective of the teaching tool used $(P<0.001)$. The significant effect was maintained at 3 months' follow-up. There was no detectable difference in nutrition knowledge post intervention or at follow-up between the two study groups. In intervention schools, younger pupils ( $8-9$ years) had better nutrition knowledge than older pupils $(10-11$ years $)(P=0.011)$.

Conclusion: This computer-based tool increases the possibilities of school-based nutrition education. If the tool's weaknesses identified during the formative evaluation are eliminated, it has the potential to make learning about nutrition more enjoyable, exciting and effective. This is of great importance considering that 'healthy' nutrition is not necessarily a topic that easily attracts pupils' attention and in view of the potential long-term health benefits of early and effective nutrition education.
\end{abstract}

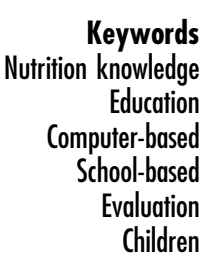

The importance of early learning of nutrition-related knowledge, attitudes and behaviours for future health has long been recognised ${ }^{1-3}$. It is well known that schoolbased nutrition education programmes play a vital role in modifying children's eating behaviour and promoting lifelong healthy eating, and numerous guidelines for schoolbased education have been published to date ${ }^{4-6}$.

The development of innovative teaching curricula in nutrition education is a continuous and demanding process. There are constantly new messages in health promotion and prevention of nutrition-related diseases, progressing theories and models in health education and behaviour, and new advances of age- and culturally appropriate teaching methodologies. Considering that children often lack interest in nutritional topics, there is a strong need for nutrition education curricula that are engaging and enjoyable for children ${ }^{7,8}$. Children's lack of interest in nutrition topics is of growing concern, because children become independent at an earlier age and start purchasing foods earlier and more frequently. Children need good nutrition knowledge and skills to help them choose from the uncountable number of food items, which are often more 'attractive' than 'nutritionally beneficial' ' Teaching tools that capture children's attention for nutrition topics are therefore essential.

Computers combine numerous communication methods, such as text, sound, still images and animation ${ }^{10}$. This increases the possibilities of developing teaching tools that are challenging, enjoyable and attractive for children, and thus computers are a valuable addition to nutrition education ${ }^{11,12}$.

The European Food Information Council has explored the world-wide web as a possible tool for communicating nutrition and health information to children and adolescents, and has created two interactive websites in four languages. One was developed for children (Cool Food Planet KIDZ) and one for adolescents (Cool Food Planet ADOLZ $)^{13}$. The websites are teaching tools for nutrition 
and lifestyle education intended for use in schools throughout Europe.

The purpose of this study was to evaluate the efficacy and feasibility of using Cool Food Planet KIDZ in a real-world environment. The strengths and weaknesses of Cool Food Planet KIDZ were explored. For the purpose of this study, the website was made available on CD-ROM.

\section{Methods}

\section{Study design and data collection}

The study design was multi-factorial with repeated measures, at three points in time, of dependent samples from control and intervention groups. The study was a school-based nutrition education intervention in which control schools used 'traditional' nutrition education materials, including worksheets, board games and card games, which were developed for the purpose of this study. Intervention schools used the Cool Food Planet KIDZ computer-based educational tool in addition to the traditional materials. Nutrition knowledge (dependent measure) was measured at baseline (t0), immediately after intervention (t1) and at 3 months' follow-up (t2) with the same instrument. Nutrition knowledge was defined by the contents of Cool Food Planet KIDZ. The questionnaire with which pupils' nutrition knowledge was determined consisted of 22 multiple-choice questions with four answer possibilities, including 'I don't know'. Pupils' demographic data, including age, gender and country of origin, were assessed. Pupils' socio-economic status was assessed using the Family Affluence Scale ${ }^{14}$ and parent's occupation, as described in detail elsewhere ${ }^{15}$. Pupils filled out the questionnaire during one 50-minute school lesson under examination conditions. Due to limited study resources, nutrition knowledge, which is obviously only one of many factors influencing nutrition behaviour, was selected as the outcome measure.

To evaluate the usability, contents and design of Cool Food Planet KIDZ, student teachers and pupils received evaluation questionnaires after the intervention. Qualitative information was collected in focus group discussions with student teachers $(n=3)$ and pupils $(n=4)$, and by observing the nutrition lessons.

\section{Selection of schools and sample size}

After study approval had been obtained from the City School Board, the selection of schools took place in collaboration with the Academy of Pedagogy of Vienna. The main criterion for selecting the schools was that they had to offer a 2-week practical experience for student teachers attending the Academy. This was decided on because student teachers have not yet 'internalised' their own teaching style, as compared with older, more experienced teachers, which was thought to reduce teaching bias. Furthermore, owing to the target group of Cool Food Planet KIDZ, the project had to be implemented in third and fourth grades, which included 8-11-year-old pupils.

The required sample size $(n=280$ pupils $)$ was calculated using the power analysis program GPOWER ${ }^{16}$. Considering an average size of 22 children per class in Vienna, at least 15 classes were required. To reduce the risk of contamination through classroom effects, each class was located in a different school. Taking non-response and non-adherence to the study protocol into account, it was decided to include a total of 20 primary schools, 10 of which were intervention schools and 10 control schools.

\section{Preparatory workshops and development of educational materials}

A one-day workshop for all participating student teachers was held by two project staff members to give an introduction to the basics in nutrition and to explain and discuss the intervention. During the workshop student teachers gave suggestions for the development of the 'traditional teaching materials' used by intervention and control schools. The traditional teaching materials included 13 worksheets, two board games and one card game. Five worksheets were developed specifically for the intervention schools, to guide the navigation through Cool Food Planet KIDZ.

A second workshop was held for the teachers who supervised the student teachers during their practical experience. The supervisors also received an introduction to the basics in nutrition and were briefed on the purpose and procedure of the intervention.

\section{Intervention}

The schools were grouped randomly into intervention and control schools by the project team. Prior to the intervention the student teachers were asked if they were comfortable with the group they were assigned to.

Student teachers had to allocate a total of 5 hours to the nutrition intervention during their 2 -week practical experience (November 2001). Due to different lesson schedules in the participating schools, the student teachers had to be given the choice whether the designated 5 hours were taught during one day or spread throughout the two weeks.

The student teachers of control schools were instructed to use only the non computer-based materials, i.e. the worksheets and games developed by the project team. In the intervention schools the student teachers were instructed to additionally have pupils use Cool Food Planet KIDZ for a total of at least 50 minutes during class time. The reason why computers were used in addition to the 'traditional' teaching tools was because only two computers are available in the majority of primary school classrooms. Pupils used the computers in pairs. Since pupils are allowed to use the computer during the break between lessons, 
pupils in the intervention schools were encouraged to use Cool Food Planet KIDZ during their break.

\section{Statistical analysis}

Data from the nutrition knowledge and evaluation questionnaires were entered twice to minimise entry bias and analysed with the Statistical Program for the Social Sciences, version 10.0 (SPSS Inc., Chicago, IL, USA). Post hoc analyses were conducted using the statistical program GPOWER to compute the effect size of the actual difference in nutrition knowledge between control and intervention groups post intervention.

Data were examined to help determine the appropriate statistical tests. Histograms, Kolmogorov-Smirnov, kurtosis and skewness were used to test the assumptions of symmetry and normality of data. Homogeneity of baseline key variables (nutrition knowledge, age, gender, grade level, country of origin and socio-economic status) within and between groups was assessed using non-parametric tests (Chi-square, Mann-Whitney $U$-test) and parametric tests (pairwise comparison, calculated in the general linear model (GLM)), as appropriate.

Only schools and pupils that adhered to the defined study protocol were included in the analysis. Owing to the coding scheme it was possible to perform the analysis using tests for dependent samples, which are more robust.
The effects and interactions of the dependent and independent variables (shown in Table 1) were calculated using the GLM for repeated measures. Levene's test was applied to test the homogeneity of variance.

The focus group discussions were transcribed and the responses coded according to developed guidelines.

\section{Results}

The response rate was quite high (86\%); however, the percentage of schools and pupils that completed the study successfully was rather small (57\%). Many pupils were lost due to incomplete questionnaires, unidentifiable coding and schools not adhering to the study protocol. A total of 13 schools (271 pupils) (consisting of eight intervention schools (145 pupils) and seven control schools (126 pupils)) from the initial 20 schools (473 pupils) successfully completed the study.

\section{Comparison of study groups}

Study groups were comparable with respect to age, gender, country of origin and grade level, as shown in Table 2.

In order to compare baseline nutrition knowledge between intervention and control schools, age groups and gender, the result of the pairwise comparison between nutrition knowledge and the variable 'group' - as

Table 1 Variables included in the general linear model

\begin{tabular}{|c|c|c|}
\hline & Variable name & Variable values \\
\hline Dependent variables (within-subject variables) & Nutrition knowledge & $\begin{array}{l}\text { Baseline knowledge (t0) } \\
\text { Post-intervention knowledge (t1) } \\
3 \text { months' follow-up knowledge (t2) }\end{array}$ \\
\hline Independent variables (between-subject factors) & $\begin{array}{l}\text { Group } \\
\text { Age } \\
\text { Gender }\end{array}$ & $\begin{array}{l}\text { Intervention } \\
\text { Control } \\
8-9 \text {-year-olds } \\
10-11 \text {-year-olds } \\
\text { Girls } \\
\text { Boys }\end{array}$ \\
\hline
\end{tabular}

Table 2 Comparison of age, gender, country of origin and grade level between pupils from intervention and control schools

\begin{tabular}{|c|c|c|c|c|c|c|c|}
\hline \multirow[b]{2}{*}{ Variable } & \multicolumn{3}{|c|}{ Intervention schools } & \multicolumn{3}{|c|}{ Control schools } & \multirow[b]{2}{*}{ Total row count } \\
\hline & Count & Column \% & Row \% & Count & Column \% & Row \% & \\
\hline 8-9-year-olds & 104 & 71.72 & 56.52 & 80 & 63.49 & 43.48 & 184 \\
\hline 10-11-year-olds & 41 & 28.28 & 47.13 & 46 & 36.51 & 52.87 & 87 \\
\hline Total column count & 145 & & & 126 & & & 271 \\
\hline Girls & 73 & 50.34 & 52.14 & 67 & 53.17 & 47.86 & 140 \\
\hline Boys & 72 & 49.66 & 54.96 & 59 & 46.83 & 45.04 & 131 \\
\hline Total column count & 145 & & & 126 & & & 271 \\
\hline Austrian & 133 & 91.72 & 54.51 & 111 & 88.10 & 45.49 & 244 \\
\hline Non Austrian & 12 & 8.28 & 44.44 & 15 & 11.90 & 55.56 & 27 \\
\hline Total column count & 145 & & & 126 & & & 271 \\
\hline Third grade & 62 & 42.76 & 58.49 & 44 & 34.92 & 41.51 & 106 \\
\hline Fourth grade & 83 & 57.24 & 50.30 & 82 & 65.08 & 49.70 & 165 \\
\hline Total column count & 145 & & & 126 & & & 271 \\
\hline
\end{tabular}


calculated in the GLM - was used. The rationale for using the pairwise comparison was to avoid conducting additional tests, which decreases the power of the test and increases type I error.

Baseline nutrition knowledge of the two study groups, age groups and genders was comparable, as shown in Table 3 .

\section{Changes in nutrition knowledge}

The results of the GLM for repeated measures showed a significant increase in nutrition knowledge in both groups from baseline to post intervention, which was maintained at 3 months' follow-up, as shown in Table 4 . The main effect of 'time' in the GLM was statistically significant $(P<0.001)$. The pairwise comparison $(P$-values adjusted with Bonferroni) showed that the overall nutrition knowledge (for intervention and control schools combined) increased significantly from a mean of 11.23 at baseline to a mean of 13.72 (standard error (SE) 0.187; $P<0.001)$ after the intervention. Nutrition knowledge at 3 months' follow-up was significantly higher than baseline nutrition knowledge in both groups (SE 0.198; $P<0.001$ ). There was no difference between the post-intervention mean and the 3 months' follow-up mean.

The main effect of the variable 'group' was not statistically significant, suggesting that the nutrition education intervention was effective in increasing nutrition knowledge irrespective of the teaching tool used.

The results of the test of within-subject effects indicated significant effects for the 'time by group by age' interaction $(P<0.05)$. The pairwise comparison showed that younger pupils (aged 8-9 years) in intervention schools had significantly higher scores on the nutrition knowledge questionnaire than did older pupils (aged 10-11 years) in intervention schools (SE $0.61 ; P=0.011$ ), as shown in Table 5.

Table 3 Comparison of baseline nutrition knowledge between intervention and control schools

\begin{tabular}{|c|c|c|c|c|c|c|c|c|c|c|}
\hline & \multicolumn{5}{|c|}{ Intervention schools } & \multicolumn{5}{|c|}{ Control schools } \\
\hline & Count & Mean* & SE† & Min‡ & Max§ & Count & Mean & SE & Min & Max \\
\hline Girls & 73 & 11.68 & 0.39 & 5 & 20 & 67 & 11.30 & 0.42 & 1 & 20 \\
\hline Boys & 72 & 11.32 & 0.38 & 5 & 20 & 59 & 10.75 & 0.39 & 4 & 18 \\
\hline 8-9-year-olds & 104 & 11.67 & 0.32 & 5 & 20 & 80 & 10.96 & 0.39 & 1 & 20 \\
\hline 10-11-year-olds & 41 & 11.07 & 0.50 & 5 & 19 & 46 & 11.17 & 0.42 & 4 & 18 \\
\hline All pupils & 145 & 11.50 & 0.27 & 5 & 20 & 126 & 11.04 & 0.29 & 1 & 20 \\
\hline
\end{tabular}

* Mean number of correct answers from a total of 22 questions.

† Standard error of the mean.

$\ddagger$ Minimum number of correct answers.

$\S$ Maximum number of correct answers.

Table 4 Nutrition knowledge at baseline, post intervention and 3 months' follow-up in intervention and control schools

\begin{tabular}{|c|c|c|c|c|c|c|c|c|}
\hline & \multicolumn{4}{|c|}{ Intervention schools $(n=145)$} & \multicolumn{4}{|c|}{ Control schools $(n=126)$} \\
\hline & Mean* & SE† & Minł & $\overline{\operatorname{Max} \S}$ & Mean & SE & Min & $\overline{\operatorname{Max}}$ \\
\hline Baseline & 11.50 & 0.27 & 5 & 20 & 11.04 & 0.29 & 1 & 20 \\
\hline Post intervention & 14.07 & 0.28 & 3 & 21 & 13.61 & 0.31 & 4 & 21 \\
\hline 3 months' follow-up & 13.59 & 0.29 & 2 & 21 & 13.51 & 0.28 & 3 & 21 \\
\hline
\end{tabular}

* Mean number of correct answers from a total of 22 questions.

† Standard error of the mean.

$\ddagger$ Minimum number of correct answers.

$\S$ Maximum number of correct answers.

Table 5 Nutrition knowledge at baseline, post intervention and 3 months' follow-up in intervention and control schools by age

\begin{tabular}{|c|c|c|c|c|c|c|c|c|}
\hline & \multicolumn{4}{|c|}{ Intervention schools } & \multicolumn{4}{|c|}{ Control schools } \\
\hline & \multicolumn{2}{|c|}{$\begin{array}{c}8-9 \text {-year-olds } \\
\quad(n=104)\end{array}$} & \multicolumn{2}{|c|}{$\begin{array}{c}10-11 \text {-year-olds } \\
(n=41)\end{array}$} & \multicolumn{2}{|c|}{$\begin{array}{l}\text { 8-9-year-olds } \\
\quad(n=80)\end{array}$} & \multicolumn{2}{|c|}{$\begin{array}{c}10-11 \text {-year-olds } \\
(n=46)\end{array}$} \\
\hline & Mean* & SE† & Mean & SE & Mean & SE & Mean & SE \\
\hline Baseline & 11.67 & 0.32 & 11.07 & 0.50 & 10.96 & 0.39 & 11.17 & 0.42 \\
\hline Post intervention & 14.21 & 0.35 & 13.71 & 0.46 & 13.95 & 0.38 & 13.02 & 0.51 \\
\hline 3 months' follow-up & 14.03 & 0.32 & 12.49 & 0.58 & 13.54 & 0.38 & 13.46 & 0.42 \\
\hline
\end{tabular}

* Mean number of correct answers from a total of 22 questions.

† Standard error of the mean. 


\section{Effect size}

The actual effect size of the difference in nutrition knowledge post intervention between intervention and control groups was calculated post hoc with the GPOWER statistical program. Using the group means of correct answers after the intervention of 14.07 for intervention schools ( $n=145$ pupils) and 13.61 for control schools ( $n=126$ pupils) and the combined standard deviation of 3.43 , the effect size of $d=0.134$ was calculated.

\section{Results of evaluation questionnaires and qualitative data}

One hundred and sixty-two pupils from intervention schools filled out the evaluation questionnaire. Most pupils gave Cool Food Planet KIDZ the grade 'very good' ( $n=95,60 \%)$ and only two pupils (1.2\%) gave the grade 'not good at all'. Fifty-nine per cent of the pupils thought it was 'very fun' to learn about nutrition with the computer and $81 \%$ indicated that they would visit Cool Food Planet KIDZ on the Internet. Children especially liked the pictures (52\%), the topics (52\%) and the moving figures (42\%). Almost all of the pupils (97\%) indicated that they wanted to work with the computer again in class and 89\% indicated that they wanted to work with the Internet.

During the focus group discussions, pupils from intervention schools were more critical about Cool Food Planet KIDZ. They loved the design and the cartoons, and the fact that they could 'click until your head smokes'. Despite the fact that pupils mentioned there was too much to read, the font was too small and that the sentence structure and the words were too complicated, younger pupils seemed more enthusiastic about Cool Food Planet KIDZ.

Interestingly, the pupils were able to describe the cartoons in detail during the focus groups, but had difficulties remembering the content. For example, most pupils knew that one of the figures helped the other to drink from a glass, but only a few remembered that it was important to 'drink at least five glasses of fluids per day'.

When asked whether they preferred working with the computer or with the teacher, the pupils replied that they like both. They said that the teacher 'explains better' and when working with the computer they 'feel in charge and more independent'.

The overall message of the evaluation questionnaires filled out by the student teachers from intervention schools was that the purpose of Cool Food Planet KIDZ, i.e. to be a teaching tool for nutrition and lifestyle education in schools, is very good. All student teachers indicated that Cool Food Planet KIDZ was a helpful teaching tool, but they mentioned that they would only consider using it again if the text was shortened and facilitated and if more interactive features were included.

The three focus group discussions with student teachers confirmed the results of the evaluation questionnaire and also revealed additional information. They mentioned that 'computers are not appropriate to generate knowledge, rather kids need to see, feel and experience things before using the computer'. However, they saw great potential in the use of computer-based education. They would combine computer-based tools with traditional teaching tools and use computers primarily to reinforce what pupils have already learned.

The observations and teaching protocols revealed that all schools spent at least 5 hours on the nutrition project. However, the content of the nutrition lessons varied between schools, despite the provision of guidelines the teachers were asked to follow. The average amount of time children from intervention schools used the computerbased tool was 30 minutes compared with the advised 50 minutes. Pupils in intervention schools did not use the computers during their break. In control schools not all of the worksheets or board and card games were used.

\section{Discussion}

A significant increase in pupils' nutrition knowledge was found in both study groups after the intervention and was maintained through follow-up. This suggests that using Cool Food Planet KIDZ in addition to traditional teaching tools (worksheets, card and board games) neither hindered nor strengthened the impact intervention had on children's nutrition knowledge.

The current body of evidence about the efficacy of using computer-based nutrition education tools is still limited. Even though the benefits of using computer-based nutrition education tools in schools is widely documented $^{17-22}$, only a few studies have compared the efficacy of teaching tools in experimental designs ${ }^{23,24}$. These schoolbased experimental studies showed a significant increase in nutrition knowledge in pupils who used computerbased tools compared with those who did not use the computer, which is in contrast to this study. However, the comparison of such experimental studies is limited, due to considerable variations in study design. The computerbased nutrition education tool was used either in addition to traditional teaching tools $\mathrm{s}^{23}$ or on its own ${ }^{24}$. Other variations include differences in age group, duration of the intervention or nutrition topics. To better compare the results of evaluation studies, it is of great importance to develop a 'gold standard' for evaluating the efficacy of computer-based nutrition education tools.

The lack of a detectable difference in nutrition knowledge between control and intervention schools in our study must be interpreted in consideration of the study's limitations and in view of the fact that this was a formative evaluation of Cool Food Planet KIDZ. Study limitations were that Cool Food Planet KIDZ was used in addition to traditional materials and that too little time was allocated to the use of computers. The advantage of conducting this evaluation during the practical experience of student teachers - which was intended to reduce 
teaching bias - turned out to be a study limitation. The student teachers were new to the class and had to become familiar with the new teaching environment. The additional challenge of administering the nutrition intervention could have contributed to the fact that the student teachers did not adhere to the teaching guidelines. This led to variations in the content of the nutrition lessons between the grades, which is another source of bias.

The effect size (Cohen's $d$ ) of the difference in nutrition knowledge after the intervention between the two study groups is 0.134 , reflecting only a minor difference in nutrition knowledge between the groups. The effect size of the difference in knowledge between study groups after an educational intervention can be used to interpret the educational impact of the intervention. It has been suggested that educationally meaningful programmes result in an effect size of 0.2 or greater ${ }^{25}$. Using this cutoff point in our study would lead to the conclusion that the educational impact of using the computer-based tool in addition to 'traditional' teaching tools was 'not meaningful'. However, it must be taken into consideration that the effect size is not only a result of the efficacy of an educational intervention. There are a number of other factors (e.g. sample size or standard deviation) that influence effect size. Comparison with effect sizes found in other studies that evaluate the efficacy of computer-based nutrition education tools would therefore enable a more valuable interpretation. Unfortunately, no reference studies that document the effect size were found.

Even if the quantitative analysis of this evaluation did not show an additional beneficial learning effect of using Cool Food Planet KIDZ, the qualitative data brought to light valuable information. Weaknesses of the teaching tool were identified and recommendations for modifying the current version of Cool Food Planet KIDZ were made. Other information obtained through the qualitative data support the use of computer-based nutrition education tools in general. Student teachers' mentioned that if nutrition education is neglected in schools, it is certainly not only due to a lack of teachers motivation or commitment. As emphasised in the focus group discussions, it is often due to teachers' time constraints or their poor nutrition knowledge. Teachers welcome 'easy to use' and interactive computer-based tools, since they reduce preparation time and do not require teachers to have strong background knowledge in nutrition. The student teachers were all in favour of using computer-based nutrition education tools in addition to traditional tools, to reinforce what pupils have already learned. The results of the focus group discussions with student teachers support the purpose of Cool Food Planet KIDZ to complement not replace - traditional materials, to increase educational possibilities, and to make learning about nutrition more enjoyable and exciting for pupils. In this respect it is of great importance to investigate further how computer-based nutrition education can optimally be combined with traditional nutrition education.

Focus groups with pupils showed that they enjoyed working with Cool Food Planet KIDZ and found it to be exciting. This was the case especially in the younger age group, including pupils aged 8 and 9 years. They also benefited more from the use of Cool Food Planet KIDZ than did older pupils, as was shown in their significantly higher nutrition knowledge post intervention and at follow-up.

This experimental study adds to the limited body of evidence describing the efficacy of computer-based nutrition education. Since the few existing studies focus solely on the transfer of nutrition knowledge, there is still a strong need for studies investigating whether or not computer-based nutrition education tools are beneficial in supporting pupils' ability to translate their acquired nutrition knowledge into nutrition behaviour.

Considering current evidence in computer-based nutrition education and the results of the qualitative data, teachers are strongly encouraged to explore computerbased education tools as a complementary teaching tool for nutrition education. The modified version of Cool Food Planet KIDZ has great potential to be a valuable teaching tool for nutrition education in schools.

\section{Acknowledgements}

The author acknowledges the support of Professor Ibrahim Elmadfa of the Institute of Nutritional Sciences, University of Vienna, Austria and Dr Barrie Margetts of the Institute of Human Nutrition, University of Southampton, UK during her doctorate studies. The European Food Information Council (EUFIC) funded the study, and the author especially thanks Ms. Anna Jung, Director of EUFIC for her kind support. The co-operation of the Academy of Pedagogy, Vienna is also appreciated. Special thanks are extended to the team at 'thepartners.at' for their support and to the student teachers and pupils who participated in this study. The help of all assistants is greatly valued.

\section{References}

1 Tones K, Tilford S. Health education in schools. In: Tones K, Tilford S, eds. Health Education: Effectiveness, Efficiency and Equity, 2nd ed. London: Chapman and Hall, 1994; $121-48$

2 Contento I, Balch GI, Bronner YL, Lytle L, Maloney SK, Olson CM, et al. The effectiveness of nutrition education and implications for nutrition education policy, programs, and research: a review of research. Journal of Nutrition Education 1995; 27: 277-419.

3 Perez-Rodrigo C, Aranceta J. School-based nutrition education: lessons learned and new perspectives. Public Health Nutrition 2001; 4(1A): 131-9.

4 World Health Organization (WHO). Nutrition: An Essential Element of a Health Promoting School. WHO Information Series on School Health, Document 4. Geneva: WHO, 1998. Available at http://www.who.ch/hpr. Accessed August 2002. 
5 Dixey R, Heindl I, Loureiro I, Pérez-Rodrigo C, Snel J, Warnking J. Healthy Eating for Young People in Europe: A School-based Nutrition Education Guide. Copenhagen: European Network of Health Promoting Schools, 1999. Available at http://europa.eu.int/comm/health/ph/ programmes/health/reports/1997/fp_promotion_1997_exs_ D2_en.pdf. Accessed August 2002.

6 Centers for Disease Control and Prevention. Guidelines for school health programs to promote lifelong healthy eating. Morbidity and Mortality Weekly Report 1996; 45(RR-9): 1-41. Available at http://www.cdc.gov/epo/mmwr/ preview/mmwrhtml/00042446.htm. Accessed August 2002.

7 Croll JK, Neumark-Sztainer D, Story M. Healthy eating: what does it mean to adolescents? Journal of Nutrition Education 2001; 33: 193-8.

8 Westenhöfer J. Ernährungsverhalten und Ernährungseinstellungen von Schülern. Paper presented at the $37 \mathrm{th}$ Scientific Congress of the German Nutrition Society, Bonn, 16-17 March 2000.

9 Tomkins A. Vitamin and mineral nutrition for the health and development of the children of Europe. Public Health Nutrition 2001; 4(1A): 101-7.

10 Beermann K. Computer-based multimedia: new directions in teaching and learning. Journal of Nutrition Education 1996; 28: 15-8.

11 Kolasa K, Miller MG. New developments in nutrition education using computer technology. Journal of Nutrition Education 1996; 28: 7-14.

12 Matheson D, Spranger K. Content analysis of the use of fantasy, challenge and curiosity in school-based nutrition education programs. Journal of Nutrition Education 2001; 33: $10-6$.

13 Cool Food Planet: Nutrition and Life-style Education Tool. Available at http://www.coolfoodplanet.org. Accessed August 2002.

14 Currie C, Elton RA, Todd J, Platt S. Indicators of socioeconomic status for adolescents: the WHO Health Behavior in School-aged Children Survey. Health Education Research 1997; 12: 385-97.
15 Kreisel K. Computer-based nutrition education in primary schools. Outcome evaluation of the program 'Cool Food Planet KIDZ' in Vienna, Austria. Doctoral thesis, Institute of Nutritional Science, University of Vienna, Vienna, 2002.

16 Erdfelder E, Faul F, Buchner A. GPOWER: a general power analysis program. Behavior Research Methods, Instruments and Computers 1996; 28: 1-11.

17 Matheson D, Achterberg C. Description of a process evaluation model for nutrition education computer-assisted instruction programs. Journal of Nutrition Education 1999; 31: $105-13$.

18 Matheson D, Achterberg C. Ecologic study of children's use of computer nutrition education program. Journal of Nutrition Education 2001; 33: 2-9.

19 DiSorga L, Glanz K. The 5 a day virtual classroom: an on-line strategy to promote healthful eating. Journal of the American Dietetic Association 2000; 100: 349-52.

20 Brown SJ, Liebermann DA, Fan YC, Wilson DM, Pasta DJ. Educational video game for juvenile diabetes: results of a controlled trial. Medical Informatics 1997; 22: 77-89.

21 Seaman C, Kirk T. Novel computer software for nutrition education. Health Education 1996; 4: 32-4.

22 McKinley M, Livingstone MBE. Dish it up! An interactive multi media CD ROM for the promotion of nutrition education in secondary school children. Personal e-mail correspondence, April 2002. Information available at http://www. foodstandards.gov.uk/science/research/NutritionResearch/ n09programme/n09projectlist/n09012/n09012results. Accessed February 2003.

23 Turnin MC, Tauber MT, Couvaras O, Jouret B, Bolzonella C, Bourgeois $\mathrm{O}$, et al. Evaluation of microcomputer nutritional teaching games in 1,876 children at school. Diabetes and Metabolism 2001; 27: 459-64.

24 Lloyd-Kolkin D. School-based nutrition education using multimedia. New Hope, PA: Health and Education Communication Consultants, 2001 [unpublished].

25 Slavin RE. A reader's guide to scientifically based research. Educational Leadership 2003; 60: 12-6. 